\title{
Long non-coding RNA RP11-468E2.5 curtails colorectal cancer cell proliferation and stimulates apoptosis via the JAK/STAT signaling pathway by targeting STAT5 and STAT6
}

Li Jiang ${ }^{1+}$, Xu-Hai Zhao ${ }^{2 \dagger}$, Yin-Ling Mao ${ }^{3 *}$, Jun-Feng Wang ${ }^{4}$, Hui-Jun Zheng ${ }^{5}$ and Qing-Shan You ${ }^{3}$

\begin{abstract}
Background: Long non-coding RNAs (IncRNAs) are tumor-associated biological molecules and have been found to be implicated in the progression of colorectal cancer (CRC). This study aims to examine the effects of IncRNA RP11468E2.5 and its target genes (STAT5 and STAT6) on the biological activities of CRC cells via the Janus kinase-signal transducer and activator of transcription (JAK/STAT) signaling pathway.

Methods: We initially screened the GEO database for differentially expressed IncRNAs related to CRC and then made a prediction of the implicated target genes. Then we collected CRC tissues and adjacent normal tissues from 169 CRC patients. Human CRC HCT116 and SW480 cells were treated with small interference RNA (siRNA) against RP11-468E2.5, AG490 (an inhibitor of the JAK/STAT signaling pathway), or both in combination. Next, we measured the effects of RP11-468E2.5 treatment on cellular activities such as cell viability, cycle distribution and cell apoptosis, and studied interactions among RP11-468E2.5, STAT5/STAT6, and the JAK/STAT signaling pathway. Finally, an in vivo tumor formation assay was performed to observe the effect of RP11-468E2.5 on tumor growth.

Results: The CRC-related gene microarray data showed low expression of RP11-468E2.5 in CRC surgical specimens. However, RP11-468E2.5 was confirmed to target STAT5 and STAT6, which participate in the JAK/STAT signaling pathway. CRC tissues showed lower expression of RP11-468E2.5, higher expression of STAT5, STAT6 and of the cell cycle marker Cyclin D1 (CCND1), compared to the findings in adjacent normal tissues. The treatment of siRNA against RP11-468E2.5 increased expression of JAK2, STAT3, STAT5, STAT6, CCND1 and BCl-2 along with the extent of STAT3, STAT5 and STAT6 phosphorylation, while lowering expression of P21 and P27. Treatment with AG490 exhibited approximately opposite effects, whereas siRNA against RP11-468E2.5 treatment stimulated CRC cell proliferation and reduced cell apoptosis, while promoting cell cycle entry; AG490 treatment reversed these results.
\end{abstract}

Conclusions: Altogether, we conclude that up-regulation of RP11-468E2.5 inhibits the JAK/STAT signaling pathway by targeting STAT5 and STAT6, thereby suppressing cell proliferation and promoting cell apoptosis in CRC.

Keywords: Long non-coding RNA RP11-468E2.5, Colorectal cancer, STAT5 gene, STAT6 gene, Janus kinasesignal transducer and activator of transcription signaling pathway, Proliferation, Apoptosis

\footnotetext{
* Correspondence: 569655212@qq.com

${ }^{+}$Li Jiang and Xu-Hai Zhao are regarded as co-first authors.

${ }^{3}$ Department of Abdominal Radiotherapy, Harbin Medical University Tumour

Hospital, No. 150, Haping Road, Nangang District, Harbin 150081, People's

Republic of China

Full list of author information is available at the end of the article
}

C The Author(s). 2019 Open Access This article is distributed under the terms of the Creative Commons Attribution 4.0 International License (http://creativecommons.org/licenses/by/4.0/), which permits unrestricted use, distribution, and reproduction in any medium, provided you give appropriate credit to the original author(s) and the source, provide a link to the Creative Commons license, and indicate if changes were made. The Creative Commons Public Domain Dedication waiver (http://creativecommons.org/publicdomain/zero/1.0/) applies to the data made available in this article, unless otherwise stated. 


\section{Background}

Colorectal cancer $(\mathrm{CRC})$ is an aggressive disease with high morbidity and mortality throughout the world [1]. Each year, more than 1 million people are affected by CRC, accompanied by overt metastatic or invasive disease. The malignant form of CRC accounts for some 600,000 deaths worldwide each year [2]. Aging, mutations, and chronic intestinal inflammation are all known factors responsible for the occurrence and progression of CRC [3]. The high rates of cancer metastasis, recurrence and emergent chemoresistance pose great obstacles to effective treatments of patients with CRC at all stages, highlighting the need for the novel improved therapeutic strategies [4].

Long non-coding RNAs (lncRNAs) have been shown to play a crucial role in the regulation of tumorigenesis, and molecular biology studies implicate abnormal expression levels of lncRNAs such as LINC00152 in the development and progression of CRC cell tumorigenesis [5]. LncRNAs also serve as regulators of gene expression in interaction with diverse mechanisms. Regulation by lncRNAs depends on its site-specific interaction with DNA, as well as on their binding to proteins and chromosomes forming protein complexes [6]. Janus kinasesignal transducer and activator of transcription (JAK/ STAT) signaling pathway is considered an important signal transduction pathway for cell development [7]. Previous studies have revealed that phosphorylated and nonphosphorylated STAT proteins are constitutively present in cytoplasm and nuclei. Other studies also proved that the dimer of phosphorylated STAT forms in the cytoplasm and then migrates into the nucleus. Only phosphorylated STAT homodimer or heterodimer species possess a DNA-binding capability. Upon combination with co-activator proteins, these species mediate transcriptional regulation $[8,9]$. Under stimulation from cytokines, the messenger signal transducer and activator of transcription-5 tyrosine phosphorylation (pY-STAT5) are transiently activated, whereas STAT5 and the promoted pY-STAT5 show persistent overexpression in multiple neoplastic cell types [10]. Moreover, there is reportedly an underlying biological interaction between different STATs, i.e. STAT5 and STAT6. This pair of proteins functions as an activator and inhibitor for gene expression, as well as a modulator of the epigenetic landscape of immune cells [11].

A previous report indicated a positive correlation between the activation of the JAK/STAT signaling pathway and colorectal adenoma progression [12]. Another previous study suggested a relationship between lncRNAs and the JAK/STAT signaling pathway, which indicated a regulatory potential in biological processes [13]. Furthermore, Mao et al. have shown that elevated phosphoSTAT5 expression is prevalent in adenocarcinoma of the colon and is associated with poor prognosis [14, 15]. Therefore, this present study aims to investigate the role of lncRNA RP11-468E2.5 on proliferation and apoptosis of CRC cells via interaction with the JAK/STAT signaling pathway and STAT5 and STAT6.

\section{Materials and methods}

\section{Ethics statement}

This study was performed with the approval from the Ethics Committee of the Harbin Medical University Tumour Hospital. All participating patients provided written informed consents. Animal experiments in this study were carried out in strict accordance with the Guide for the Care and Use of Laboratory animals published by the US National Institutes of Health.

\section{Microarray-based gene expression profiling}

The Gene Expression Omnibus (GEO) database (http://www. ncbi.nlm.nih.gov/geo) was used to download CRC-related microarray expression data (GSE4107 and GSE21510) and annotate probe files. Background correction and normalization of each data microarray were processed by the Affy installation package of $\mathrm{R}$ software [16]. The empirical Baysian method of the linear model in the Limma installation package was combined with a traditional $t$-test to filter out nonspecific expression data, and then screen out the differentially expressed lncRNAs [17], which were predicted by the Multi Experiment Matrix website (MEM, http://biit.cs.ut.ee/ $\mathrm{mem} /$ ). The target gene was analyzed by Kyoto Encyclopedia of Genes and Genomes (KEGG) pathway enrichment analysis using the WebGestalt database (http://www.webgestalt.org) to confirm the major biochemical metabolic pathways and signaling pathways of the target gene [18].

\section{Study subjects}

Following procedures in previous studies [14, 15], we collected CRC and adjacent normal tissue samples from 169 patients with CRC (95 males and 74 females; mean age 57 years, range 44 to 72 years), obtained by surgical resection at the Harbin Medical University Tumour Hospital. Patients had no pre-surgical treatments, such as chemotherapy, radiotherapy or biotherapy or other therapies. A follow-up monitoring was conducted on all patients until April 30, 2016. The resected primary tumor specimen was evaluated using the tumor node metastasis (TNM) staging system (American Joint Committee on Cancer (AJCC), version 6.0) [19]. The degree of differentiation of the tumor was evaluated according to the classification of tumors from the World Health Organization (WHO); accordingly, the tumors were categorized into undifferentiated, poorly differentiated, moderately differentiated, and well differentiated tumors. The pathological diagnosis [20] was confirmed by two senior pathologists. 


\section{Reverse transcription quantitative polymerase chain reaction (RT-qPCR)}

The total RNA of tissues was extracted according to the instructions provided in the Trizol kit (NO. 15596-018; Invitrogen Inc., Carlsbad, CA, USA). We then undertook reverse transcription of the extracted RNA to complementary DNA (cDNA), again following manufacturer's instructions of the reagent kit (Thermo Fisher Scientific Inc., Waltham, MA, USA) using a two-step method. The obtained cDNA was stored at $-80^{\circ} \mathrm{C}$ until use in the subsequent experiments. RT-qPCR was conducted using the TaqMan probe method, following the instructions of the reagent kit (KR011A1; Bejing Puyihua Science and Technology Co., Ltd., Bejing, China). The primer sequences are shown in Table 1. Glyceraldehyde-3-phosphate dehydrogenase (GAPDH) was used as an internal reference of gene expression. The relative mRNA expression of the target gene was expressed as $2^{-\Delta \Delta \mathrm{Ct}}$. The experiments were repeated three times [21]. This method was also used for the mRNA detection in the subsequent cell experiments.

\section{Immunohistochemistry}

The tissues were fixed in $10 \%$ neutral formalin, dehydrated, embedded in paraffin, cut into $5 \mu \mathrm{m}$ serial sections, and dehydrated. The tissue sections were then treated with $3 \%$ hydrogen peroxide at room temperature for 10 min in order to block endogenous peroxidase activity, after which the sections were blocked with normal nonimmunone serum for $10 \mathrm{~min}$. The sections were then incubated with primary rabbit polyclonal antibodies against phosphorylated (p)-STAT5 (Tyr694/699, SC-81524, Santa Cruz Biotechnology, Inc., Santa Cruz, CA, USA) at a dilution of 1:100, p-STAT6 (Tyr64, SC-136019, Santa Cruz Biotechnology, Inc., Santa Cruz, CA, USA) at a dilution of 1:200, and Cyclin D1 (CCND1) at $4{ }^{\circ} \mathrm{C}$ overnight. Sections were then incubated with horseradish peroxidase-labeled secondary antibody goat anti-rabbit immunoglobulin G (IgG) diluted to $1: 500$ at $37^{\circ} \mathrm{C}$ for $20 \mathrm{~min}$, followed by incubation with $50 \mu \mathrm{l}$ of streptavidin peroxidase solution at room temperature for $10 \mathrm{~min}$ and final development with diaminobenzidine (DAB) solution. After counterstaining with hematoxylin, all sections were dehydrated, cleared, and mounted for microscopic examination, with phosphate buffered saline (PBS) solution as negative control (NC). We evaluated the protein positive expression as follows: five visual fields of tumor area at high magnification $(\times 400)$ were randomly selected from each section. The positive expression rate was determined according to the percentage of positive cells to the total number of cells. The density of positive cells was determined according to the semi-quantitative assessment with reference to the percentage ratio of positive cells. In the event that the ratio was less than $15 \%$, the protein expression was deemed negative (-); when the ratio ranged from 15 to $25 \%$, the protein expression was weakly positive (+); 25 to 50 was moderately positive $(++)$, ratio $50-75 \%$ was strongly positive $(+++)$, and a ratio exceeding $75 \%$ indicated that protein expression was significantly and strongly positive $(++++)$. The sections were classified into two groups according to the staining results: sections with a ratio of $\mathrm{p}$ STAT5 and p-STAT6 positive cells $<15 \%$ were assigned to the negative group, and sections with a corresponding ratio of exceeding 15\% were assigned to the positive group. In the case of CCND1, sections with a ratio of CCND1 positive cells less than $5 \%$ were deemed negative, and sections with the ratio exceeding $5 \%$ were deemed positive.

\section{Cell culture}

We used the following cell lines provided by the Heilongiiang Tumor Institute: (1) human CPC cell lines of RKO, poorly-differentiated colon cancer cell line, (2) LOVO, a cell line first isolated in 1971 from a segment of metastatic tumor nodule in a 56-year-old Caucasian male colon cancer patient, (3) SW620, which was isolated from a lymph node from a 51-year-old Caucasian patient with blood group $\mathrm{A}, \mathrm{Rh}+$ ), (4) SW480, isolated from a 50-year-old Caucasian male with primary colon cancer, and (5) HCT116, which has a nearly diploid stemline chromosome number, with a modal number of 45 chromosomes (62\%) and polyploids occurring in $6.8 \%$ of cells. Cells were incubated at $37^{\circ} \mathrm{C}$ with $5 \% \mathrm{CO}_{2}$ in Roswell Park Memorial Institute (RPMI) 1640 culture medium containing 15\% fetal bovine serum (FBS) (Santa Cruz Biotechnology, Inc., Santa Cruz, CA, USA).

\section{Establishment of RNA interference (RNAi) vector}

The sequences of RP11-468E2.5 small interference RNA (siRNA) were designed using the Ambion siRNA design software (Austin, TX, USA), with the TTCAAGAGA sequence in the loop structure, and employing the GPSupersilencing Vector. The lentivirus-based packaging system (L110424; Shanghai Beinuo Biotechnology Co., Ltd., Shanghai, China) consisted of the following four kinds of plasmids: pRsv-REV, p MDlg-pRRE, pMD2G, and an interfering plasmid.

\section{Cell grouping and transfection}

HCT116 and SW480 cells in the logarithmic growth phase were seeded into a six-well culture plates at a cell density of $4 \times 10^{5}$ cells per well. After cell confluence reached 80 to $90 \%$, we conducted X-tremeGENE siRNA transfection. The transfection reagents were mixed and left to stand for $20 \mathrm{~min}$ before transfer into the six-well culture plates. After transfection, cells were incubated at $37^{\circ} \mathrm{C}$ with $5 \% \mathrm{CO}_{2}$ and saturated humidity for $48 \mathrm{~h}$. After replacing the culture medium, the cells were incubated for 24 to $48 \mathrm{~h}$. Each cell line was assigned to the 
blank (without any transfection), NC (transfected with NC sequence for siRNA against RP11-468E2.5), silncRNA (transfected with siRNA sequence against RP11-468E2.5), AG490 (treated with AG490, the inhibitor of the JAK/STAT signaling pathway), and AG490 + si-lncRNA groups (co-treated with siRNA sequence against RP11-468E2.5 and AG490).

\section{Western blot analysis}

The collected cells were treated with $100 \mu \mathrm{l}$ of radioimmunoprecipitation assay (RIPA) lysis buffer (R0020; Beijing Solarbio Science \& Technology Co., Ltd., Beijing, China) containing $1 \mathrm{mM}$ phenylmethylsulphonyl fluoride (PMSF) to extract the proteins, after which the protein concentration was measured using a bicinchoninic acid (BCA) kit (AR0146; Wuhan Boster Biological Technology Ltd., Wuhan, Hubei, China). The proteins were then separated by $10 \%$ polyacrylamide gel electrophoresis (PAGE), and transferred onto a polyvinylidene difluoride (PVDF) membrane (P2438; SigmaAldrich Chemical Company, St Louis MO, USA). Next, the membrane was blocked with addition of $5 \%$ bovine serum albumin (BSA), followed by incubation at room temperature for $1 \mathrm{~h}$. The membranes were then incubated with diluted primary antibodies at $4{ }^{\circ} \mathrm{C}$ overnight: JAK2 (ab108596), STAT3 (ab68153), STAT5 (ab16276), p-STAT5 (ab98338), STAT6 (ab32520), p-STAT6 (ab28829), CCND1 (ab16663), B-cell lymphoma 2 (Bcl-2) (ab32124), P21 (ab109520), and P27 (ab171091). All above antibodies were purchased from Abcam Inc. (Cambridge, MA, USA). Next morning, the membranes were washed three times $(5 \mathrm{~min}$ each) in Trisbuffered saline Tween-20 (TBST), and then incubated at room temperature for $1 \mathrm{~h}$ with the corresponding secondary anti-rabbit antibody (1:2000; ab6721; Abcam Inc., Cambridge, MA, USA). After three rinses ( $5 \mathrm{~min}$ each) in TBST, the immunocomplexes on the membrane were visualized using enhanced chemiluminescence (ECL) reagent and Gel Imager (Gel Doc EZ Imager; Bio-Rad Laboratories, Hercules, CA, USA). GAPDH (1:10000; ab181602; Abcam Inc., Cambridge, MA, USA) was used as an internal reference, with the relative expression of protein was expressed as the gray value of the target protein to that of the internal reference. Image J software was used to analyze the gray value intensity of the bands of target proteins.

\section{RNA pull-down assay}

Cells were probed for RNA expression with RP11468E2.5 labeled by $50 \mathrm{nM}$ biotin, collected, and washed using PBS. The cells were incubated in lysis buffer (Ambion, Austin, TX, USA) for $10 \mathrm{~min}$, followed by the addition of streptavidin-coated agarose bead (Invitrogen, Carlsbad, CA, USA) for further culture at room temperature for $1 \mathrm{hr}$. After washing the beads with PBS, proteins were harvested and analyzed by western blot analysis.

\section{RNA binding protein immunoprecipitation (RIP)}

The binding interactions of RP11-468E2.5 with STAT5 or STAT6 protein were detected using the RIP kit (Millipore Inc., Bedford, MA, USA). Briefly, after a pre-cooled PBS wash, the supernatant was discarded. Then, cells were lysed with lysis buffer and centrifuged at $4{ }^{\circ} \mathrm{C}$ and $12,000 \mathrm{~g}$ for $10 \mathrm{~min}$. A small amount of supernatant was taken as an input, and the remainder was coprecipitated with antibodies. Specifically, the beads were washed in PBS and resuspended in $100 \mu$ RIP wash buffer. To each group of samples, we added $5 \mu \mathrm{g}$ diluted antibodies against STAT5 (1:100; ab36153; Abcam Inc., Shanghai, China), STAT6 (1:80; ab32520; Abcam Inc., Shanghai, China), or IgG (1:1000; ab172730; Abcam Inc., Shanghai, China). Here, the IgG group served as NC. The bead-antibody complex was then washed and resuspended in $900 \mu \mathrm{l}$ RIP wash buffer, followed by incubation with $100 \mu \mathrm{l}$ cell extract overnight at $4{ }^{\circ} \mathrm{C}$. The samples were then placed on a permanent magnetic base to collect the magnetic bead-protein complexes. RNA was extracted after digestion of the samples and of the total input sample with proteinase $\mathrm{K}$ prior to further PCR quantitation.

\section{3-(4,5-dimethylthiazol-2-yl)-2,5-diphenyl-tetrazolium bromide (MTT) assay}

When cell confluence reached approximately $80 \%$, HCT116 and SW480 cells were washed twice with PBS and detached with $0.25 \%$ trypsin to prepare a single cell suspension. Cells were counted and seeded in 96-well plates, with $200 \mu \mathrm{l}$ volumes containing 3 to $6 \times 10^{3}$ cells per well. Six wells were established for each group, and after a 48-h incubation, $20 \mu \mathrm{l}$ MTT solution was added to each well (A2776-1 g; $5 \mathrm{mg} / \mathrm{ml}$; Shanghai Shifeng Biological Technology Co., Ltd., Shanghai, China) and incubated for an additional $4 \mathrm{~h}$. The culture medium was discarded, and $150 \mu \mathrm{l}$ dimethylsulfoxide (DMSO) was added to each well, followed by gentle agitation for 10 min. An enzyme-linked immunosorbent assay (ELISA) reader was used to measure the optical density (OD) values at a wavelength of $490 \mathrm{~nm}$ in each well at 12, 24 and $48 \mathrm{~h}$. Then a curve of cell viability was plotted, with triplicate repetitions of the experiment.

\section{Propidium iodide (PI) staining}

Cells were collected and centrifuged, and the supernatant was discarded. Cells were then recentrifuged at $1200 \mathrm{rpm}$ at $4{ }^{\circ} \mathrm{C}$. After being rinsed twice with cooled PBS, the cells were fixed with pre-cooled $70 \%$ ethanol at $4{ }^{\circ} \mathrm{C}$ overnight. The following day, cells were collected, centrifuged, washed with pre-cooled PBS, and resuspended. Following the re-suspension, $100 \mu$ portions were added and mixed with RNase to obtain a final concentration of $1 \mathrm{mg} / \mathrm{ml}$, and left in a water bath at $3{ }^{\circ} \mathrm{C}$ 
for $30 \mathrm{~min}$. After the addition of PI staining solution to obtain a final concentration of $50 \mu \mathrm{g} / \mathrm{ml}$, cells were stained at $4{ }^{\circ} \mathrm{C}$, in subdued light for $40 \mathrm{~min}$ and rinsed with PBS. PI was detected at a wavelength $>575 \mathrm{~nm}$ and the percentage of cells at each stage of the cycle was calculated.

\section{Annexin V-fluorescein isothiocyanate (FITC)/PI double staining}

An annexin V-FITC/PI double staining reagent kit (556, 547; Shanghai Solja Technology Co., Ltd., Shanghai, China) was used to analyze the rate of cell apoptosis. Cells from all groups were centrifuged at $2000 \mathrm{rpm}$ for 5 $\mathrm{min}$, and then resuspended and centrifuged again at 200 $\mathrm{rpm}$ for 5 to $10 \mathrm{~min}$. Cells were washed suspended in $300 \mu$ liluted binding buffer and then incubated with $5 \mu \mathrm{l}$ Annexin V-FITC in subdued light at room temperature for $15 \mathrm{~min}$. Cells were then placed in an ice bath in the dark for $5 \mathrm{~min}$ along with $5 \mu \mathrm{l}$ PI staining solution prior to conducting flow cytometry analysis. Flow cytometry was conducted to determine FITC concentration at excitation wavelengths of 480 and $530 \mathrm{~nm}$ and PI at an excitation wavelength $>575 \mathrm{~nm}$.

\section{Immunofluorescence assay}

The cell slides were treated with polylysine at room temperature for $1 \mathrm{~h}$ and rinsed three times with sterile water ( 5 min each), followed by a 30-min ultraviolet irradiation period. The slides were placed into 12-well plates, which were washed twice with PBS, whereupon the wells were seeded with CRC cell lines (HCT116 and SW480). Allowing $48 \mathrm{~h}$ for adherence of the cells to the chamber walls, cells were fixed in $4 \%$ paraformaldehyde for $20 \mathrm{~min}$ at room temperature and immersed three times $(5 \mathrm{~min}$ each) in pre-cooled PBS. Next, cells were blocked with the addition of $3 \% \mathrm{BSA} /$ phosphate buffered saline with Tween-20 (PBST) at room temperature for $1 \mathrm{~h}$, incubated with 3\% BSA/PBST-diluted primary antibody (p-STAT5, 1:200; p-STAT6, 1:200) at $4{ }^{\circ} \mathrm{C}$ overnight, and next morning rinsed three times with PBS. Subsequently, cells were incubated with 3\% BSA/PBST-diluted secondary antibody in subdued light for $1 \mathrm{~h}$, rinsed three times with precooled PBS, and then incubated with $0.1 \mu \mathrm{g} / \mathrm{ml}$ 4'6-diamidino-2-phenylindole (DAPI) for $1 \mathrm{~min}$. After being rinsed three times in ice cold PBS, cells were sealed and imaged with a microscope.

\section{In vivo tumor formation assay}

A total of $30 \mathrm{BALB} / \mathrm{c}$ nude mice (aged from 4 to 6 weeks; weighed $17 \mathrm{~g}$ to $20 \mathrm{~g}$ ) were purchased from SLAC Laboratory Animal Co., Ltd. (Changsha, China) and housed in a specific pathogen-free environment. The nude mice were then subcutaneously injected with $2 \times$ $10^{7}$ cells of the types mentioned above. Afterwards, the mice were euthanized, and the tumors extracted, photographed, and measured with a vernier caliper. Tumor volume was calculated with the formula $\left(a \times b^{2}\right) / 2$, where a represented the shorter axis and $\mathrm{b}$ the longer axis. We then plotted the mean volume at each time point $(n=6$ in each group).

\section{Statistical analysis}

SPSS 21.0 software (IBM Corp., Armonk, NY, USA) was applied for data analyses. The measurement data were expressed as mean \pm standard deviation. One-way analysis of variance (ANOVA) was used for multiple group comparisons. Comparisons between two groups were conducted by $t$-test. $p<0.05$ was considered statistically significant.

\section{Results}

\section{LncRNA expression profiles in CRC}

To search for differentially expressed lncRNAs in CRC, two lncRNA-related microarray data entries were retrieved from the GEO database for CRC. The GSE4107 is a dataset for early onset CRC, which includes 22 samples, ten of which are normal and 12 are CRC. Comparatively, GSE21510 consists of a total of 148 samples, which includes 25 normal and 123 CRC samples. Microarray data GSE4107 and GSE21510 related to CRC were analyzed using $\mathrm{R}$ language, and the top ten lncRNAs with the most differential expression in both microarrays were profiled (Fig. 1a b). The analysis of microarray data showed that among the top ten IncRNAs, only RP11-468E2.5 had low expression levels in CRC (Fig. 1c). Therefore, we used RP11-468E2.5 for this study.

\section{STAT5 and STAT6 are target genes of RP11-468E2.5}

With the microarray-based gene expression profiled, the next step was to examine the target genes of RP11468E2.5. To do so, the MEM website was used to assist in predicting the target genes of lncRNA. The results showed that STAT5 and STAT6 were target genes of RP11468E2.5, such that both proteins contributed to regulation of the JAK/STAT signaling pathway (Table 2).

\section{RP11-468E2.5 is negatively correlated with the JAK/STAT signaling pathway-related genes}

RT-qPCR was employed to determine expression of RP11-468E2.5 and the JAK/STAT signaling pathwayrelated genes in CRC tissues. When compared to the adjacent normal tissues, CRC tissues showed lower expression of RP11-468E2.5 and higher mRNA expression of STAT5, STAT6 and CCND1 (all $p<0.05$ ) (Fig. 2a). Pearson correlation analysis suggested that there was a negative correlation between the expression of RP11-468E2.5 and that of STAT5, STAT6, and CCND1 (Fig. 2b-d). 


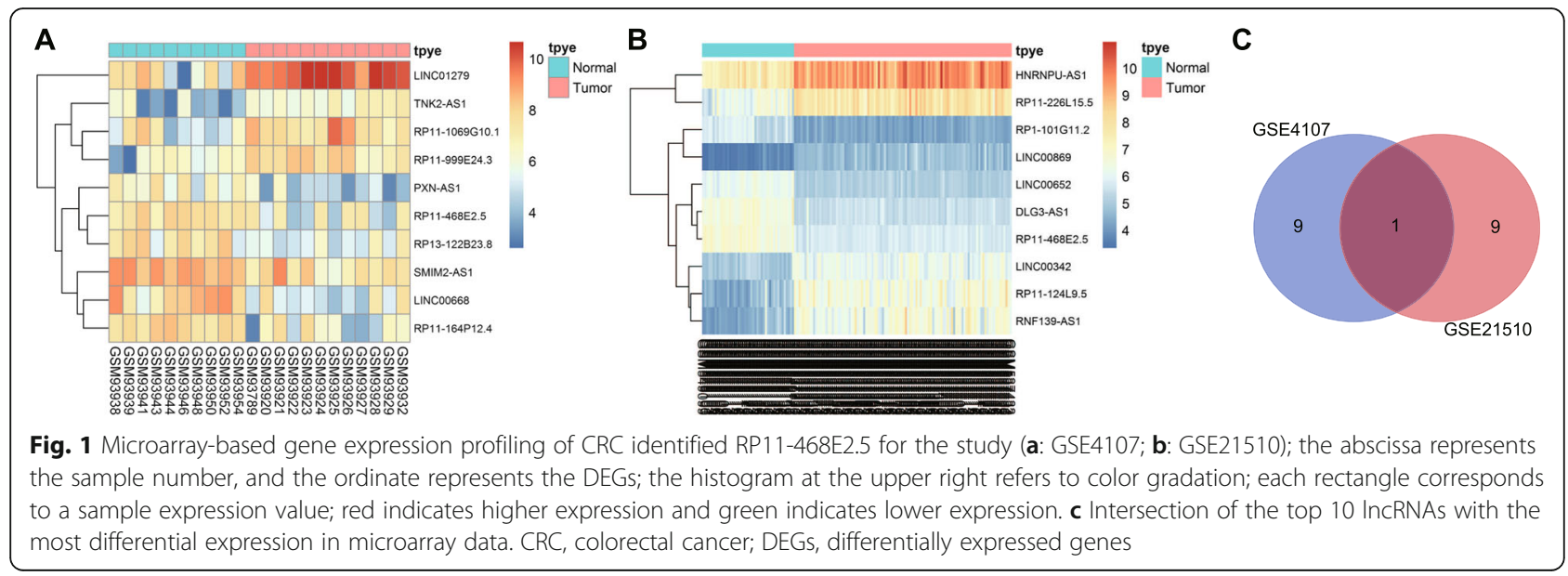

\section{CRC tissues show increased positive expression of $p$ -} STAT5, p-STAT6 and CCND1 proteins

We used immunohistochemistry (SP method) to measure the extent of STAT5/STAT6 phosphorylation and the expression of CCND1 proteins in CRC tissues and adjacent normal tissues. The results here showed a distinct cell morphology, with the extent of STAT5/ STAT6 phosphorylation and the expression of CCND1 presenting as brownish-yellow and brown pellets in the nucleus or cytoplasm. In comparison to adjacent normal tissues, the positive expression of $\mathrm{p}$ STAT5, p-STAT6 and CCND1 proteins was higher in CRC tissues (all $p<0.05$ ) (Fig. 3a). We stained the proteins using normal goat serum instead of the primary antibody, which served as NC. The specific staining of p-STAT5 and p-STAT6 was negative after blocking peptide treatment, indicating high specificity of the two antibodies (Fig. 3b).

In CRC tissues, the positive expression rate of $\mathrm{p}$ STAT5 was $47.9 \%(81 / 169)$, and that of CCND1 was
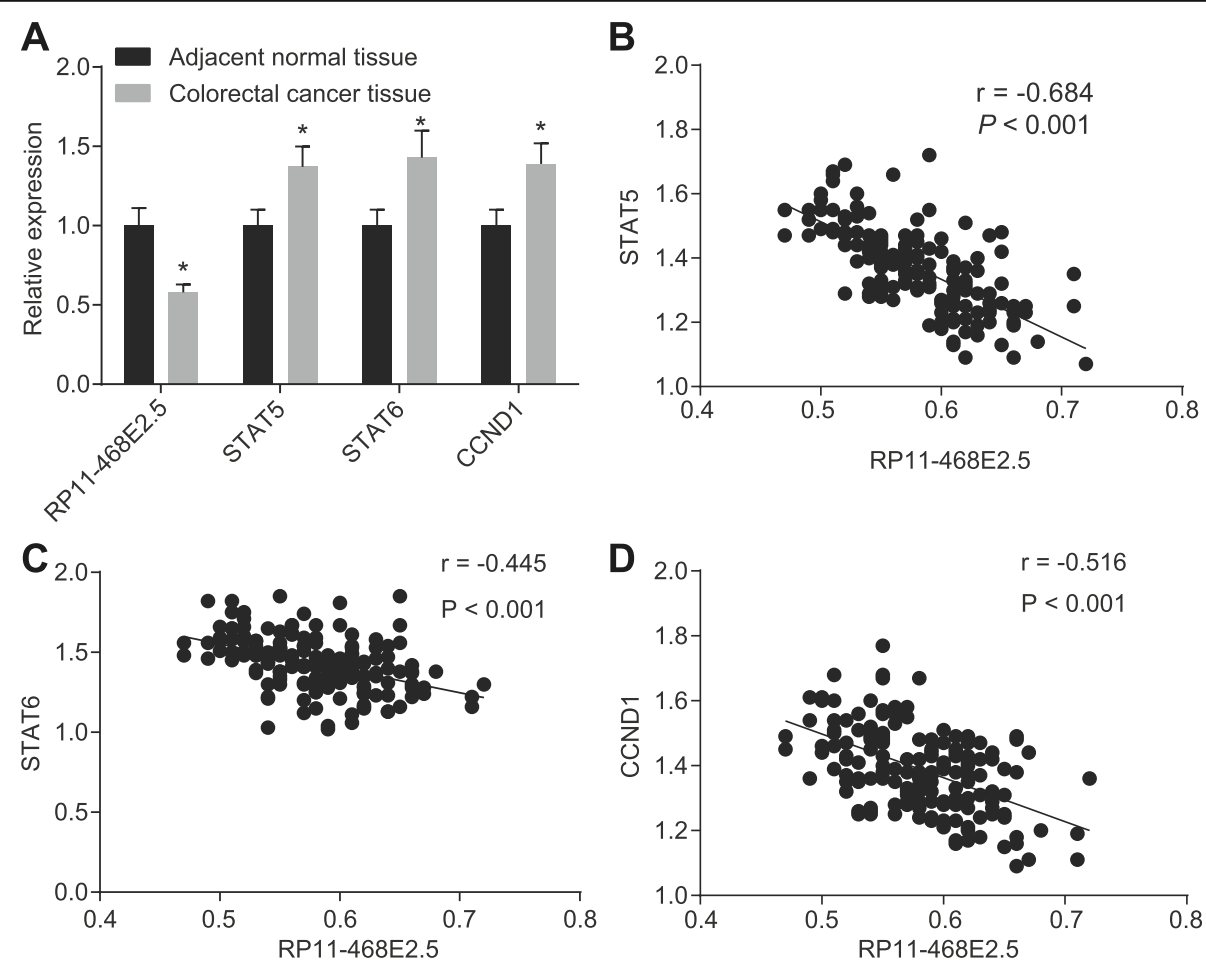

Fig. 2 RP11-468E2.5 and the JAK/STAT signaling pathway-related genes are negatively correlated in CRC tissues. A, The expression of RP11-468E2.5, STAT5, STAT6 and CCND1 detected by RT-qPCR. B, The correlation between RP11-468E2.5 with STAT5, STAT6 and CCND1 using Pearson correlation analysis. JAK, janus kinase; IncRNA, long non-coding RNA; RT-qPCR, reverse transcription quantitative polymerase chain reaction; STAT5, signal transducer and activator of transcription-5; STAT6, signal transducer and activator of transcription-6; CCND1, Cyclin D1; CRC, colorectal cancer. ${ }^{*} p<0.05$, compared with the adjacent normal tissues 


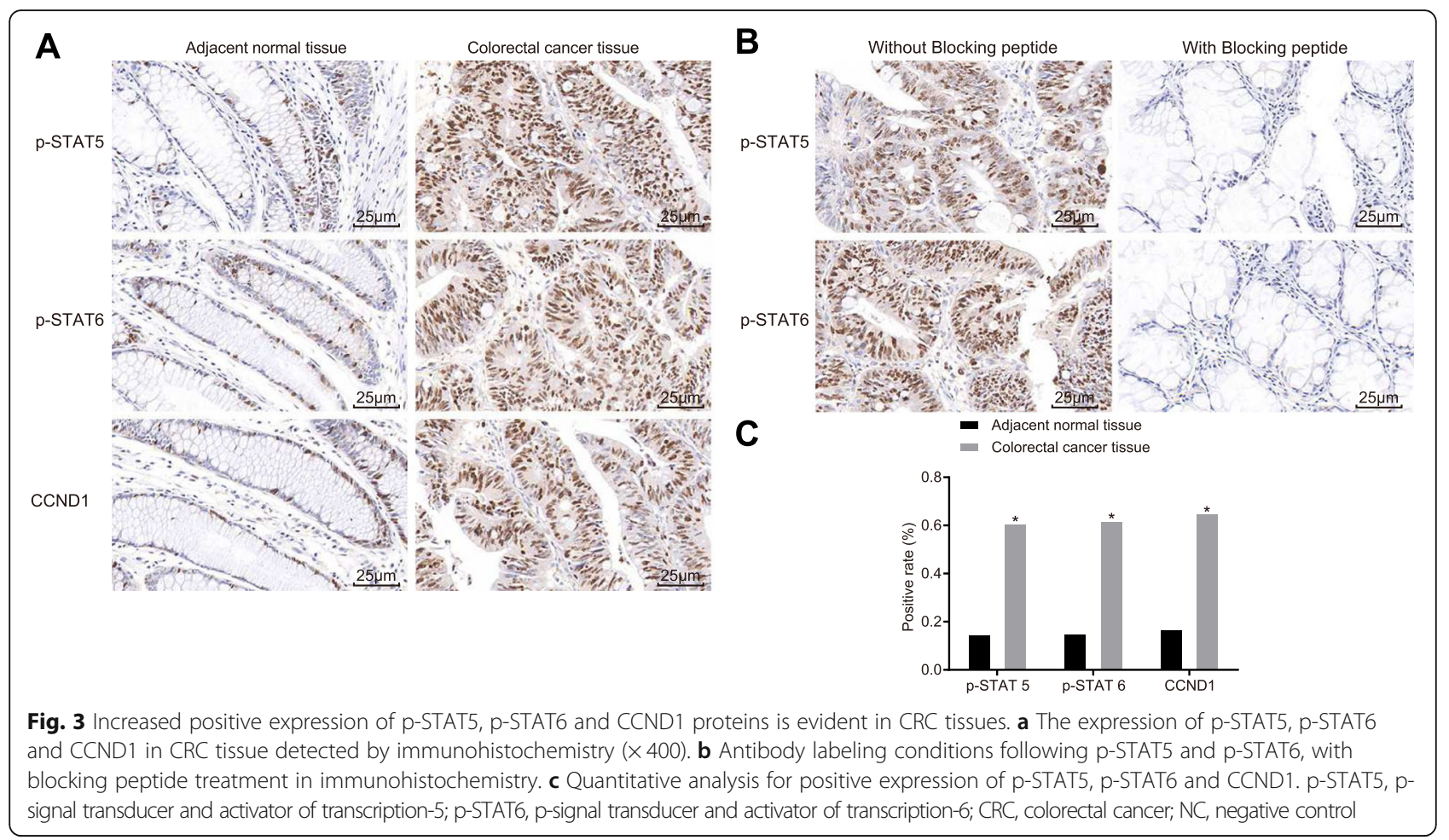

$56.2 \%$ (95/169). Pearson correlation analysis showed a positive correlation between the expression of the two proteins $(\mathrm{r}=0.727, p<0.001)$. There was also a positive correlation between the expression of p-STAT6 and CCND1. The positive expression rate of p-STAT6 was $50.9 \%(86 / 169)$ and that of CCND1 was $56.2 \%(95 / 169)$ $(\mathrm{r}=0.731, p<0.001)$ (Table 3).

The extent of STAT5/STAT6 phosphorylation is related to the tumor infiltration depth of patients with CRC

Following the CRC tissue analysis, we made a correlation analysis between p-STAT5, p-STAT6 and CCND1 protein expression and the clinicopathological features in our database of patients with CRC. Among the 169 patients with CRC, there were 81 cases with a positive expression of p-STAT5 (47.93\%), and 88 cases of negative expression of p-STAT5 (52.07\%). There were furthermore 86 cases with positive expression of p-STAT6 $(50.88 \%)$ and 83 cases with negative expression of pSTAT6 (49.11\%), and 95 cases with positive expression of CCND1 (56.21\%) and 74 cases with a negative expression of CCND1 (43.78\%) (Table 3 and 4). A subgroup analysis of p-STAT5 and p-STAT6 protein expression was performed, along with clinicopathological features in the CRC patients, i.e. age, gender, tumor size, tumor site, differentiation degree, infiltration depth, lymphatic metastasis and distant metastasis. The results of this analysis showed a positive correlation between p-STAT5 and p-STAT6 protein expression and infiltration depth
(T stage) $(p=0.002)$. There was no correlation of the proteins with the other covariates, i.e. age, gender, tumor size, tumor site, differentiation degree, lymphatic metastasis and distant metastasis (all $p>0.05$ ). We found that CCND1 protein expression was not correlated with the age, gender, tumor size, tumor site, differentiation degree, infiltration depth ( $\mathrm{T}$ stage), lymphatic metastasis or distant metastasis (all $p>0.05$ ) (Table 4).

Furthermore, we conducted subgroup analysis on RP11468E2.5 expression and the clinicopathological features as above among the 169 patients with CRC (Table 4). The results demonstrated that RP11-468E2.5 expression was correlated positively with $\mathrm{T}$ stage $(p=0.039)$ and distant metastasis $(p=0.011)$ but had no significant correlation with age, gender, tumor size, site, differentiation or lymph node metastasis $(p>0.05)$. Therefore, in compiling the above results, we infer that the tumor infiltration depth of CRC patients must correlate positively with the extent of STAT5/STAT6 phosphorylation.

\section{Highly expressed STAT5 and STAT6 are found in LOVO, SW620, SW480 and HCT116 cell lines}

We used Western blot analysis to measure protein expression of STAT5 and STAT6 in CRC cell lines (RKO, LOVO, SW620, SW480 and HCT116). As depicted in Fig. 4, the protein expression of STAT5, STAT6 and the extent of STAT5 and STAT6 phosphorylation in LOVO, SW620, SW480 and HCT116 cell lines were higher when compared to that in RKO cell lines (all $p<0.05$ ). Among the tumor 

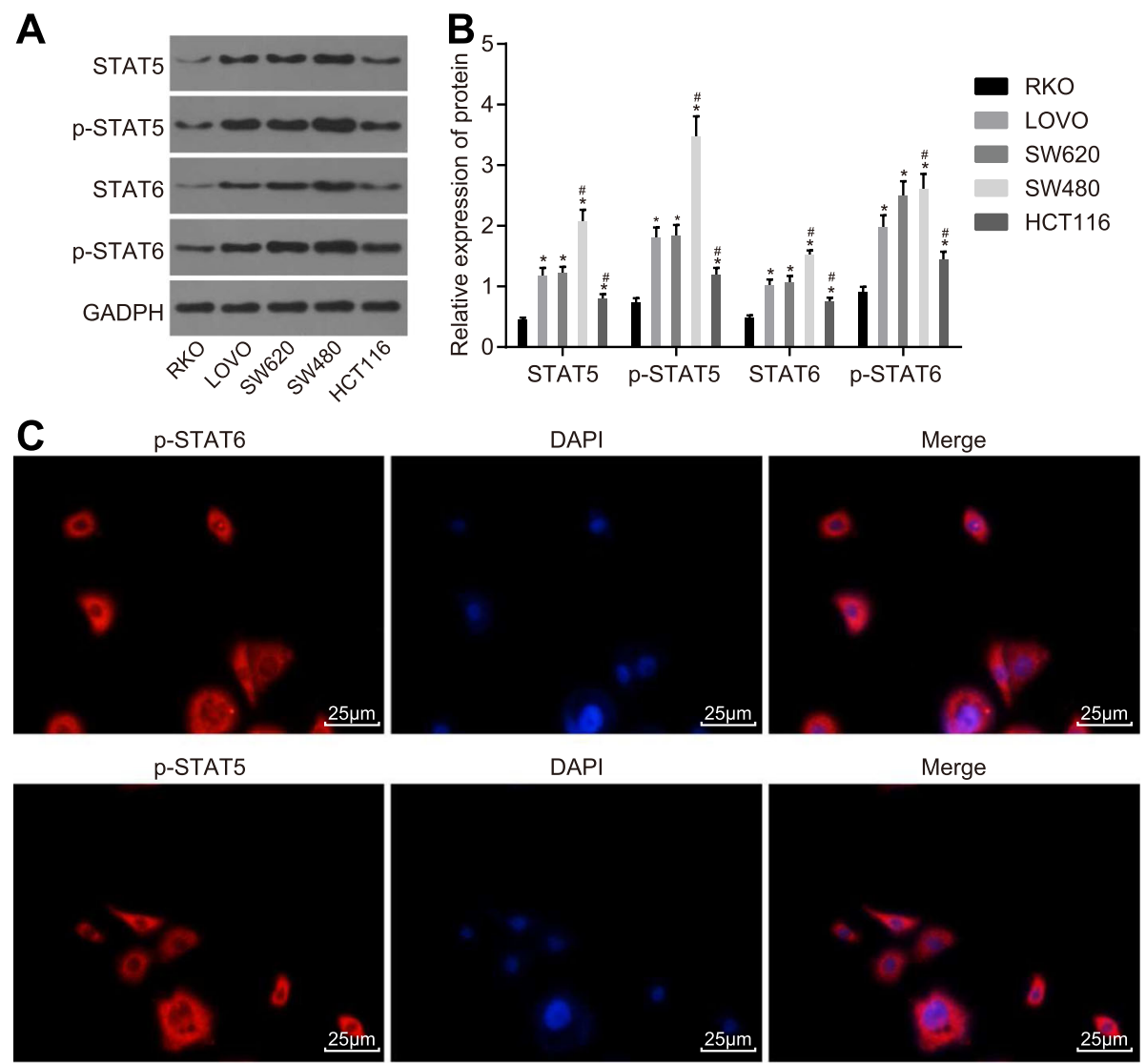

DAPI

Merge
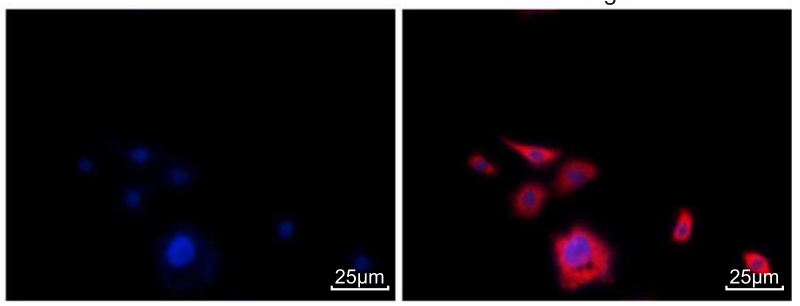

Fig. 4 Increased protein expression of STAT5 and STAT6 is found in LOVO, SW620, SW480 and HCT116 cell lines. A and B, Western blot analysis of STAT5, p-STAT5 STAT6, p-STAT6 and GAPDH proteins in different cell lines. C, Subcellular localization of p-STAT5/6 in CRC cells detected by immunofluorescence assay. p-STAT6, p-signal transducer and activator of transcription-6; STAT6, signal transducer and activator of transcription-6; p-STAT5, p-signal transducer and activator of transcription-5; STAT5, signal transducer and activator of transcription-5; GAPDH: glyceraldehyde-3phosphate dehydrogenase. * $p<0.05$, compared with RKO cell line; \# $p<0.05$, compared with LOVO cell line

cells, the SW480 cell lines in particular showed the highest expression of all proteins (all $p<0.05$ ). Therefore, we selected HCT116 and SW480 cell lines for subsequent experiments due to their generally lower metastasis and higher protein expression, respectively; we did not proceed with SW620 and LOVO cell lines because of their high metastasis. In addition, the results of subcellular localization of p-STAT5/6 in CRC cells detected by immunofluorescence assay indicated cytoplasmic retention in CRC cells, which was in accordance with immunohistochemistry results.

Effects of RP11-468E2.5 on mRNA expression of the JAK/ STAT signaling pathway- and apoptosis-related genes in HCT116 and SW480 cells

We applied RT-qPCR to determine the expression of RP11-468E2.5 and mRNA expression of JAK/STAT signaling pathway- and apoptosis-related genes in HCT116 and SW480 cells after transfection. The results (Fig. 5) showed no significant differences in mRNA expression of JAK1, STAT3, STAT5, STAT6, CCND1, Bcl-2, P21 and P27 in HCT116 and SW480 cells in the blank, NC, and
AG490 + si-lncRNA groups (all $p>0.05$ ). When compared to the blank group, we found that the AG490 + si-lncRNA group showed lower RP11-468E2.5 expression $(p<0.05)$. Comparatively, the si-lncRNA group showed much higher mRNA expression of JAK2, STAT3, STAT5, STAT6, CCND1 and Bcl-2, but relatively lower mRNA expression of P21 and P27, and RP11-468E2.5 expression, while the expression pattern tended to be opposite in the AG490 group (all $p<0.05$ ). From these findings, we inferred that up-regulated RP11-468E2.5 could decrease the mRNA expression of JAK2, STAT3, STAT5, STAT6, CCND1 and Bcl-2, but increase that of P21 and P27.

Effects of RP11-468E2.5 on protein expression of the JAK/ STAT signaling pathway- and apoptosis-related factors in HCT116 and SW480 cells

We used a Western blot analysis to determine the protein expression of the JAK/STAT signaling pathway- and apoptosis-related genes after transfection. The results (Fig. 6) showed no significant differences in protein expression of JAK1, STAT3, STAT5, STAT6, CCND1, Bcl-2, P21 and P27 

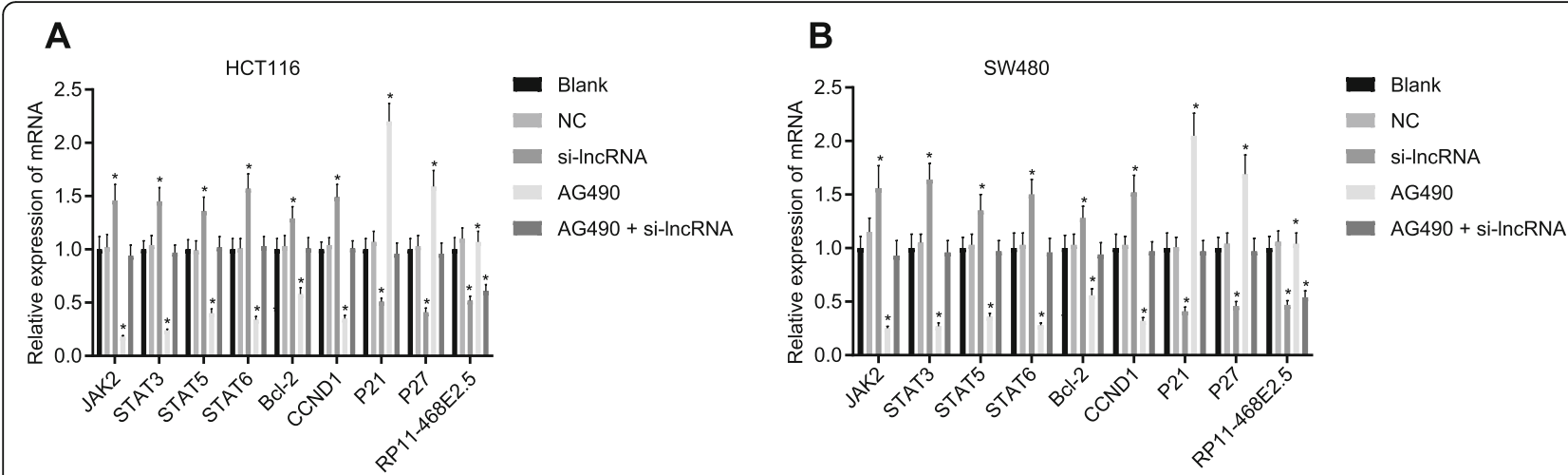

Fig. 5 Effect of RP11-468E2.5 on the mRNA expression of JAK/STAT signaling pathway- and apoptosis-related genes in HCT116 and SW480 cells. a RP11-468E2.5 expression and mRNA expression of the JAK/STAT signaling pathway-related genes and apoptosis-related genes determined by RT-qPCR in HCT116 cells. b RP11468E2.5 expression and mRNA expression of the JAK/STAT signaling pathway- and apoptosis-related genes determined by RT-qPCR in SW480 cells; RT-qPCR, reverse transcription quantitative polymerase chain reaction; NC, negative control; JAK1, janus kinase 1; STAT3, signal transducer and activator of transcription-3; STAT5, signal transducer and activator of transcription-5; STAT6, signal transducer and activator of transcription-6; BCl-2, B-cell leukemia/lymphoma 2; CCND1, Cyclin D1. ${ }^{*} p<0.05$, compared with the blank group

as well as extent of STAT6, STAT3 and STAT5 phosphorylation in HCT116 and SW480 cells in the blank, NC, and AG490 + si-lncRNA groups (all $p>0.05$ ). When compared to the blank group, there was an upward trend in the protein expression of JAK2, STAT3, STAT5, STAT6, CCND1 and $\mathrm{Bcl}-2$, while a downward trend in P21 and P27 protein expression in the si-lncRNA group $(p<0.05)$. Furthermore, the AG490 group exhibited an opposite trend as compared with the si-lncRNA group (all $p<0.05$ ). These findings indicated that up-regulated RP11-468E2.5 could decrease the protein expression of JAK2, STAT3, STAT5, STAT6, CCND1 and Bcl-2 along with extent of STAT6, STAT3 and STAT5 phosphorylation, but increase that of P21 and P27.

\section{An interaction exists among RP11-468E2.5, STAT5 and STAT6}

To further identify the possible interaction among RP11468E2.5, STAT5 and STAT6, we performed RNA pull-down and RIP assays. Based on the RNA pull-down results, STAT5 and STAT6 were enriched in RP11-468E2.5 wild type (WT) animals in contrast to RP11-468E2.5 mutant type (MUT) and NC (Fig. 7a). Meanwhile, according to RIP detection of the combination of RP11-468E2.5, STAT5, and STAT6 proteins (Fig. 7b), there was increased binding of RP11-468E2.5 to STAT5 and STAT6, as compared to IgG $(p<0.05)$. These results revealed an interaction between RP11-468E2.5, STAT5 and STAT6.

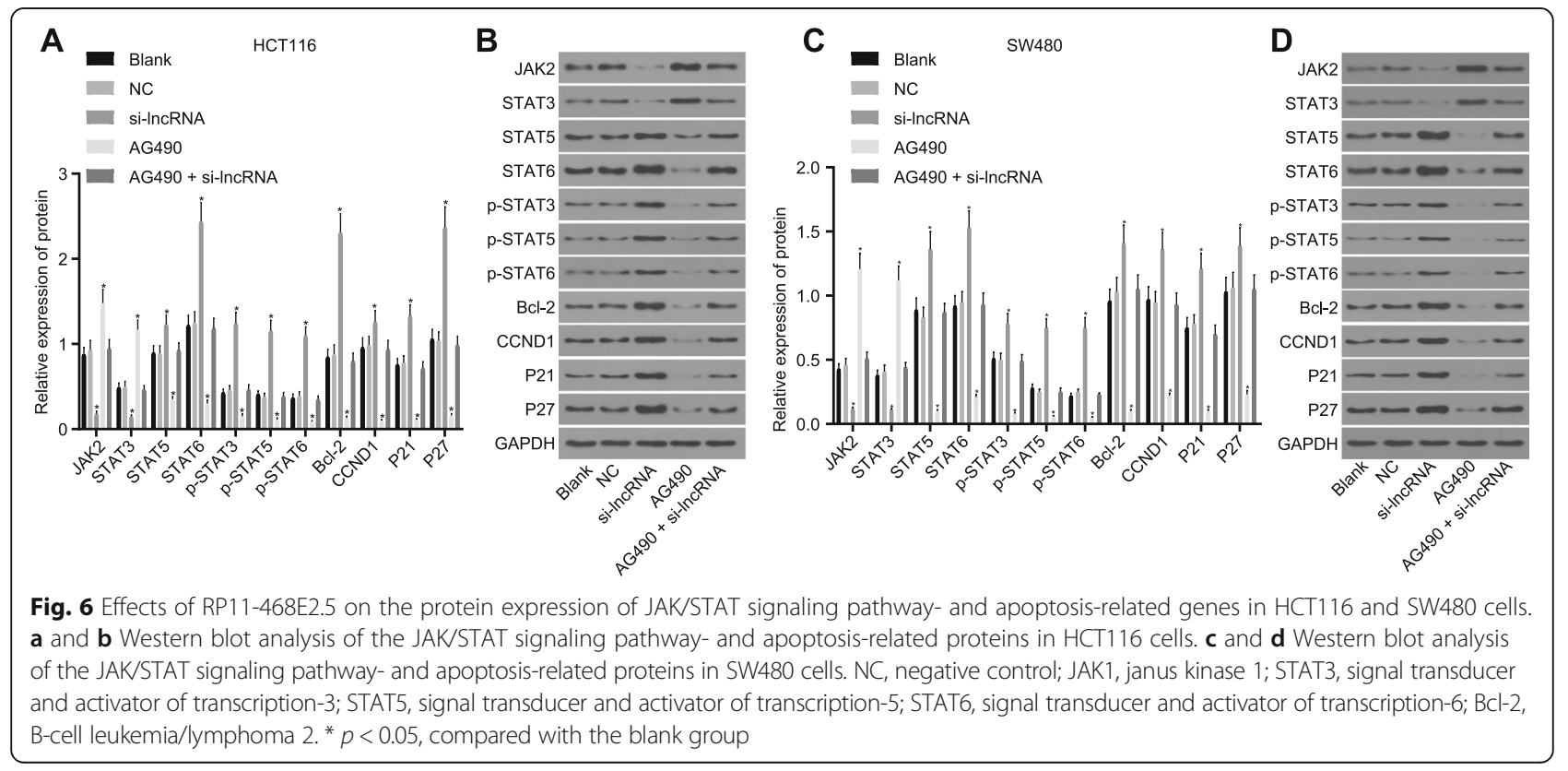



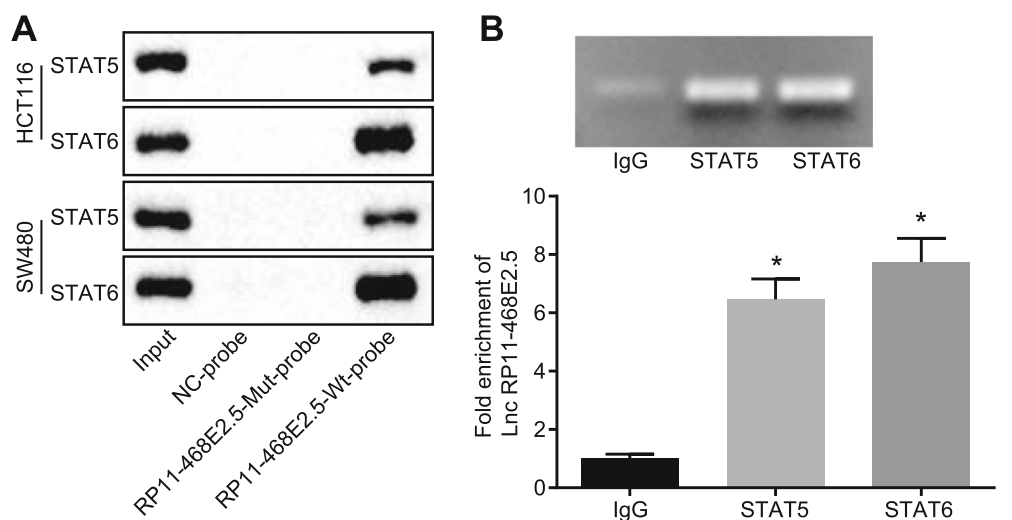

Fig. 7 RP11-468E2.5 interacts with STAT5 and STAT6. a Interaction among RP11-468E2.5, STAT5 and STAT6 detected by RNA pull-down assay. $\mathbf{b}$ Interaction among RP11-468E2.5, STAT5 and STAT6 verified using RIP assay. STAT5, signal transducer and activator of transcription-5; STAT6, signal transducer and activator of transcription- $6 .{ }^{*} p<0.05$, compared with IgG group

\section{Up-regulation of RP11-468E2.5 inhibits proliferation of HCT116 and SW480 cells}

With the effects of RP11-468E2.5 on protein expression of the JAK/STAT signaling pathway now fully examined, the next step in this project was to observe the effect of RP11468E2.5 up-regulation on proliferation of HCT116 and SW480 cells. To this end we employed an MTT assay to detect cell proliferation. The results suggested that, relative to the blank group, the si-lncRNA group showed an elevated cell vitality $(p<0.05)$, whereas the AG490 group exhibited a reduced vitality $(p<0.05)$. There were no differences in cell vitality among the NC, AG490+si-lncRNA and blank groups (all $p>0.05$ ) (Fig. 8). Therefore, we concluded that increased RP11-468E2.5 repressed the proliferation ability of HCT116 and SW480 cells.

\section{Up-regulation of RP11-468E2.5 increases HCT116 and SW480 cells arrested in G1/G0 phase}

Cell cycle distribution of HCT116 and SW480 cells in each group was assessed by PI staining. As shown in Fig. 9, there was no difference in cell cycle distribution among the blank, $\mathrm{NC}$ and AG490 + si-lncRNA groups (all $p>0.05$ ). The proliferation ability of HCT116 and SW480 cells in the AG490 group was relatively attenuated, with more cells arrested in the G1/G0 phase $(p<0.05)$. Furthermore, there were fewer cells arrested at $\mathrm{S}$ phase and $\mathrm{G} 2$ phase when compared to the blank group (all $p<0.05$ ). In the si-lncRNA group, we found fewer cells arrested at the G1/G0 phase, but increased numbers arrested at $\mathrm{S}$ phase and G2 phase (all $p<0.05$ ). These results indicated that up-regulated RP11-468E2.5 arrested cells in the $\mathrm{G} 1 / \mathrm{G} 0$ phase.
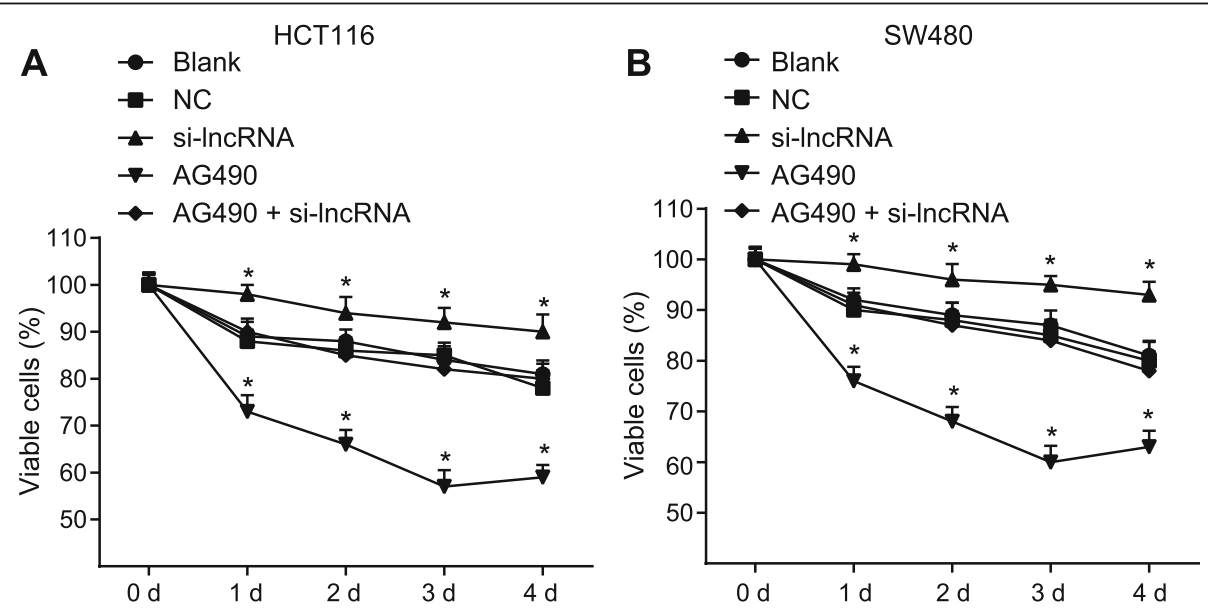

Fig. 8 Up-regulation of RP11-468E2.5 inhibits proliferation of HCT116 and SW480 cells. a Cell proliferation of HCT116 cells determined by MTT assay. $\mathbf{b}$ Cell proliferation of SW480 cells determined by MTT assay. MTT, 3-(4,5-dimethylthiazol-2-yl)-2,5-diphenyl-tetrazolium bromide; NC, negative control. * $p<0.05$, compared with the blank group 


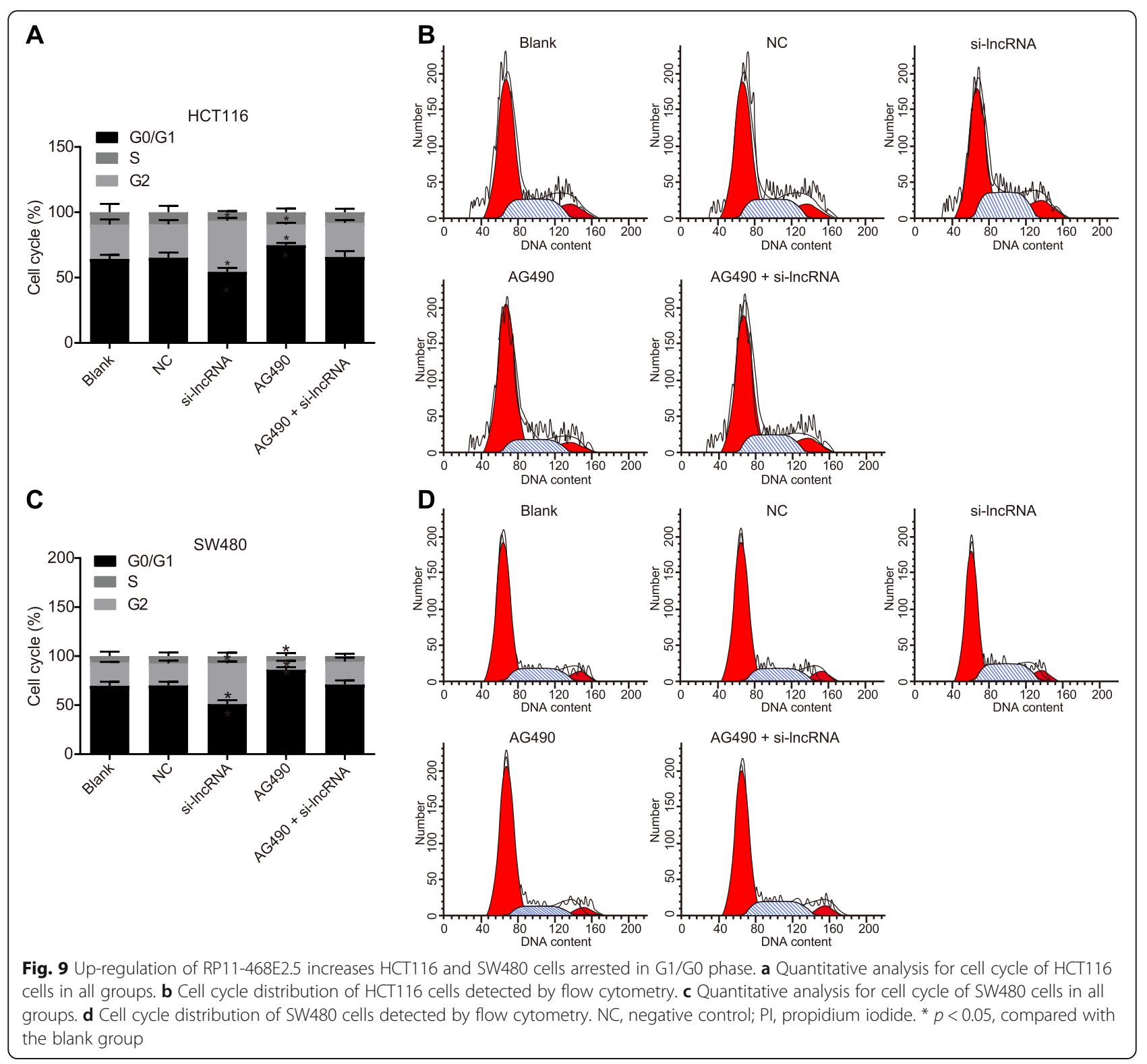

\section{Up-regulation of RP11-468E2.5 promotes apoptosis of HCT116 and SW480 cells}

To determine the effects of up-regulation of RP11468E2.5 on the apoptosis rate of HCT116 and SW480 cells in each group, we performed Annexin V-FITC/PI double staining. There was no significant difference in the apoptosis rates during the early and late stages among the blank, NC and AG490 + si-lncRNA groups (all $p>0.05$ ). The AG490 group exhibited a higher apoptosis rate during the early and late stages when compared to the blank group (all $p<0.05$ ), while the si-lncRNA group showed a lower apoptosis rate during both early and late stages (all $p<0.05$ ) (Fig. 10). Therefore, we concluded that upregulated RP11-468E2.5 could enhance the apoptosis of HCT116 and SW480 cells.
RP11-468E2.5 silencing stimulates the growth of tumors Finally, we conducted xenograft tumor assay to investigate the effect of RP11-468E2.5 on tumor growth in vivo. Here, results suggested that the tumors treated with siRNA against RP11-468E2.5 had the greatest volume and weight, while tumors with AG490 treatment had the smallest $(p<0.05)$. The mice receiving cotreatment of AG490 and RP11-468E2.5 silencing showed no evident difference in tumor growth as compared with that in mice without any treatment $(p>0.05)$ (Fig. 11).

\section{Discussion}

The prognosis of CRC patients remains poor [22]. In recent years, lncRNAs have been found to play a role in controlling gene expression and to regulate cell 


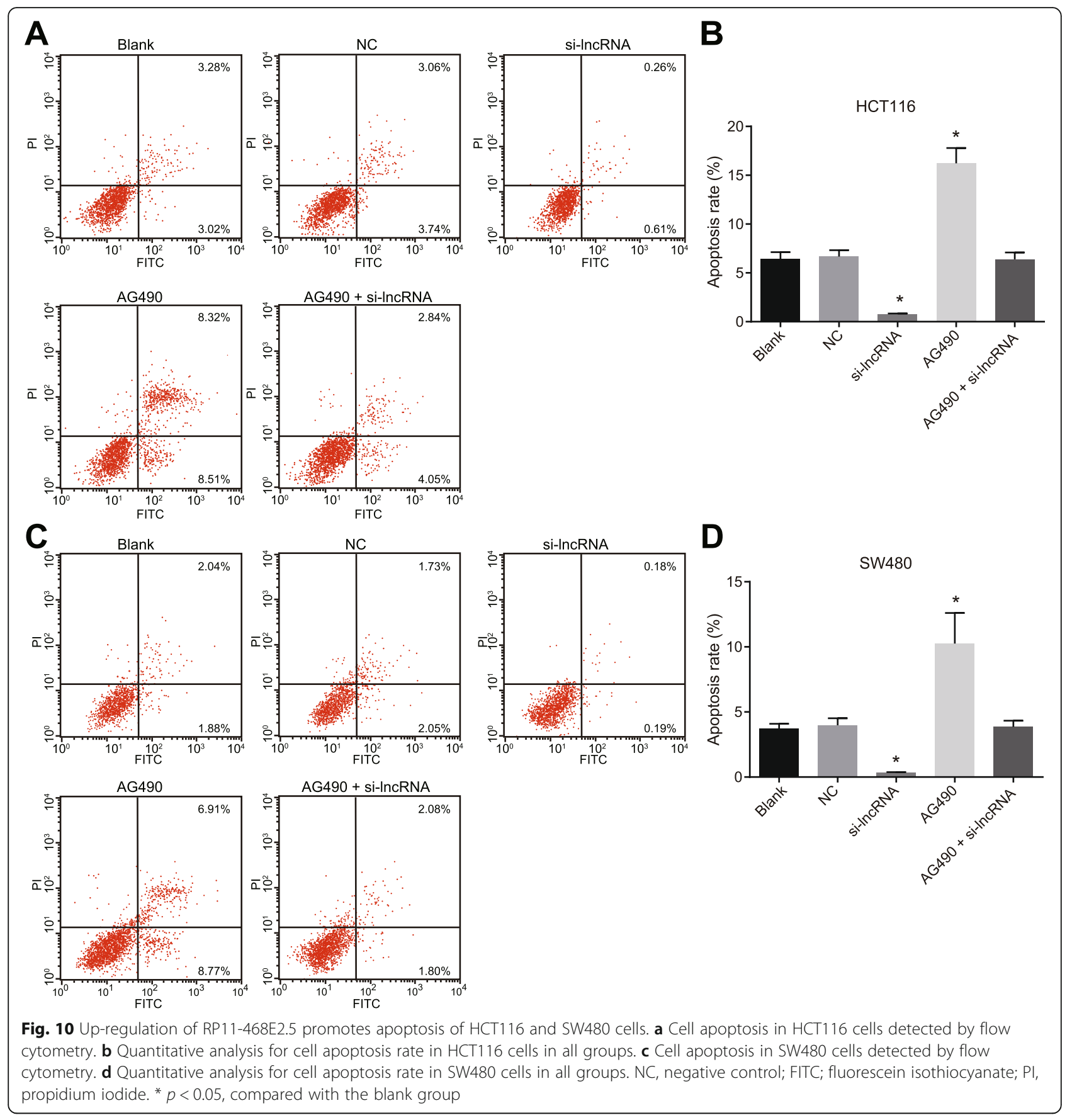

development, cell migration, cell proliferation and cell apoptosis in carcinomas [23]. In addition to this mechanism, the JAK/STAT signaling pathway also plays an important role in cellular processes and immune function [24]. Therefore, the aim of this study was to investigate the potential effects of RP11-468E2.5 and its target genes, STAT5 and STAT6 on CRC cell biological functions via the JAK/STAT signaling pathway. Our results consistently showed that high RP11-468E2.5 expression inhibits proliferation and promotes apoptosis of CRC cells by negatively regulating STAT5 and STAT6 expression via inactivating the JAK/STAT signaling pathway.

In cancers, a large part of nuclear transcription belongs to lncRNAs, and these molecules are involved in regulating many tumor markers. However, only a small number of lncRNAs have been studied in CRC, and the function of most lncRNAs in CRC remains to be established. Huang et al. indicated that low expression of lncRNA HOXB-AS3 in CRC can encode a small peptide segment, which induces inhibition of PKM shear in its 


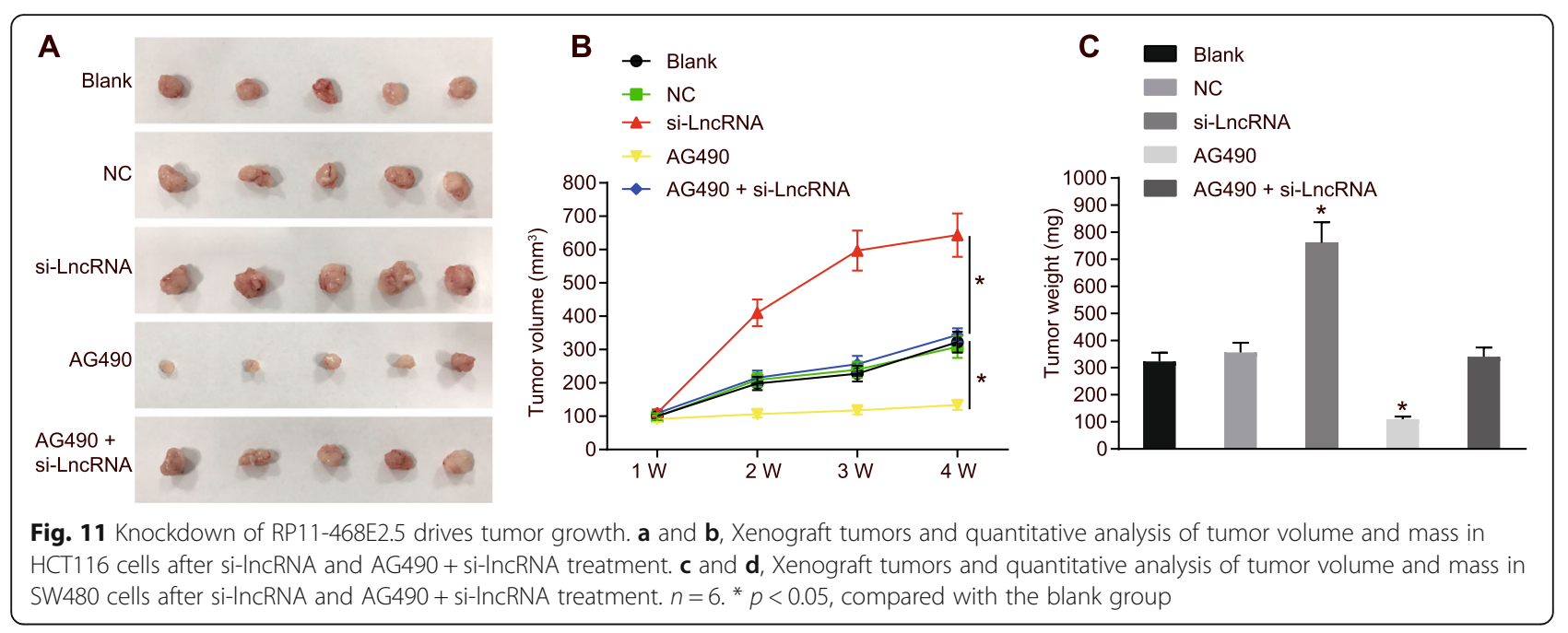

downstream metabolic recombination process, thereby inhibiting tumor growth [25]. SNHGS is an upregulated cytoplasmic lncRNA in CRC, which promotes resistance to chemotherapy with oxaliplatin by preventing STAU1 degradation and stabilizing downstream target genes [26]. In the present study, CRC tissues showed decreased expression of RP11-468E2.5 and increased expression of STAT5, STAT6 and CCND1 compared to adjacent normal tissues. LncRNAs can interact with proteins to participate in regulation of gene expression and therefore aggravate cancer progression [27]. Two members of the STAT protein family, STAT5 and STAT6, have been regarded as critical transcription factors that translocate into the nucleus upon phosphorylation by receptorassociated kinases. Upon activation of those receptors, phosphorylated STAT proteins can regulate nuclear gene expression, and interact with lncRNAs, which can play significant roles in cellular development and tumor progression [28]. In addition to the tumor progression, CCND1 cyclin, cyclin-dependent kinases and cyclindependent kinase inhibitors are well-known regulators of cell cycle transition in CRC $[29,30]$. There have been indications that the expression of protein coding genes is mediated by their promoter-sharing lncRNA. For example, knockdown via lncRNA by RNAi leads to up-regulation of the expression of protein-coding genes [31]. Previous studies have demonstrated that IncRNA RP11-462C24.1 expression is lower in CRC tissues [32]. Based on this information, we predicted that RP11-468E2.5 is expressed at a low concentration in CRC tissues. When taken together, it was concluded that lower RP11-468E2.5 expression could potentially up-regulate STAT5, STAT6, and CCND1, which made a great difference in facilitating the development and progression of CRC.

Present results show that STAT5 and STAT6 are among the target genes of RP11-468E2.5. When compared to the blank group, the si-lncRNA group showed significantly higher expression of JAK1, STAT3/5/6, CCND1 and Bcl-2, and decreased expression of P21 and P27, while the AG490 group exhibited the opposite trend. Besides, based on our RNA pull-down experiment result, WT RP11-468E2.5 obviously enhanced STAT5 and STAT6 expression in contrast to MUT RP11$468 \mathrm{E} 2.5$ or the relevant NC. The RIP assay also verified that RP11-468E2.5 was able to bind to more STAT5 and STAT6 than the IgG control did. Both of these experiments confirm that RP11-468E2.5 interacts with STAT5 and STAT6. Indeed, a previous study has demonstrated that STAT proteins can regulate JAK/STAT signaling pathway related-genes such as STAT5 [33]. According to another previous study, CCND1 and Bcl-2 concentrations are closely related to unfavorable prognosis and low survival rate of patients with pancreatic cancer. The suppression of the STAT3 signaling pathway, which targets both CCND1 and Bcl-2, led to down-regulation of the expression of both proteins, thereby inducing cellular apoptosis in cancer cells [34]. Moreover, lncRNAs participated in the suppression of STAT-3 dephosphorylation in the JAK/STAT signaling pathway, thereby acting as a regulator of cell development and cell growth [35]. The activation of STAT5 also up-regulates downstream target genes including Bcl-xL, cyclinD1/D2 and c-myc, all of which promote esophageal carcinoma cell proliferation, cell survival and immune system evasion [36]. Therefore, we feel confident in inferring that upregulation of RP11-468E2.5 negatively targeted STAT5/ 6 , and thus played an important role in the expression of the JAK/STAT signaling pathway related-genes and apoptosis related-genes in our CRC cells.

The clinical data in Table 4 showed that the expression of p-STAT5 or p-STAT6 was positively correlated with infiltration depth ( $\mathrm{T}$ stage) in $\mathrm{CRC}$, but not with other pathological parameters including tumor size. In addition, there was no significant correlation between 
CCND1 expression and all pathological parameters including tumor size. More importantly, RP11-468E2.5 expression also only correlated with T stage $(p=0.039)$ and distant metastasis $(p=0.011)$, but no with tumor size ( $p>$ $0.05)$. STAT5 has been demonstrated to assist IL-23 and thus might only promote the metastasis of CRC [37]. Also, the expression of STAT6 is correlated with lymph node metastasis and distant metastasis $(p=0.001$ and $p=0.016$, respectively) [38]. In addition, up-regulation of RP11$468 \mathrm{E} 2.5$ results in a significant decrease in the expression of STAT5, STAT6 and CCND1 as well as the extent of STAT5 and STAT6 phosphorylation in CRC cells [39]. These findings suggested that RP11-468E2.5 and pSTAT5 or p-STAT6 might be associated with CRC metastasis and infiltration while exerting little effect on the growth of CRC.

This study also demonstrated that up-regulation of RP11-468E2.5 and inhibition of the JAK/STAT signaling pathway via negatively targeting STAT5 and STAT6 suppressed proliferation and promoted apoptosis of CRC cells. As shown by the nude mouse in vivo tumor formation assay, silencing of RP11-468E2.5 led to the largest tumor growth in vivo, while the treatment with employment of AG490 resulted in slowest tumor growth, proving that down-regulated RP11-468E2.5 boosted CRC cell proliferation and inhibited apoptosis. Indeed, patients with a lower RP11-462C24.1 expression have a worse prognosis along with reduced survival rates, when compared to those with higher RP11-462C24.1 expression levels. These individuals have exhibited reduced cell proliferation, migration and invasion, and promoted apoptosis in CRC cells [32], which is consistent with the present study. Furthermore, the JAK/STAT signaling pathway is proposed to be an indispensable regulator of immune function, cell migration, and apoptosis, where its inhibition has been correlated to the severity of, for example, pancreatic cancer [40]. STAT5 also plays an important role in the maintenance of intracellular organelles as well as regulating cell proliferation, cell differentiation and survival in progenitor $\mathrm{B}$ and $\mathrm{T}$ cells $[28,41]$. Other findings have documented a significant correlation between the development and progression of CRC with components of JAK/STAT signaling pathway such as JAK1, JAK2, and STAT3 [42].

Based on microarray analysis, RP11-468E2.5 was poorly-expressed in CRC samples. The RP11-468E2.5targeted JAK/STAT signaling pathway could promote CRC cell proliferation and inhibit cell apoptosis. STAT affected cell growth or differentiation by regulating cyclin [43]. There is also a report that STAT5 expression was observed in -674-CCND1 [44]. Since CCND1, CCND2, p21 WAF/Cip1, p27 $7^{\mathrm{kip}}$ and anti-apoptotic genes such as Bcl-xl and Bcl-2 are considered to lie downstream to the JAK/STAT signaling pathway [45], their genetic expression was only detected in cellular experiments. Therefore, the role of RP11-468E2.5 in clinical studies remained to be explored and confirmed. Herein, we mainly investigated the effects of RP11468E2.5 on the JAK/STAT signaling pathway. Inhibition of JAK2 activity by treatment with AG490 significantly altered expression of STAT3/5/6. However, there remains much to be learned about how the specific inhibition of STAT5/6 expression affects CRC cell growth.

This study may draw attention to STAT5/6 as a novel therapeutic target for future CRC treatment. However, we noted some limitations of the present results. For example, the role of p-STAT5/p-STAT6 in tumor cell migration and invasion is still unknown, and further investigations on their function are necessary. Besides, the specific and detailed mechanism by which RP11-468E2.5 negatively targets STAT5 and STAT6 remains largely unclear. As reported in a prior work, lncRNAs could competitively bind to miRNAs to regulate the level of downstream genes [46]. Further research efforts in this direction are strongly recommended.

\section{Conclusions}

In conclusion, this study demonstrated that up-regulated RP11-468E2.5 resulted in inhibited cell proliferation and promoted cell apoptosis in CRC. This effect was obtained via down-regulating STAT5 and STAT6 expression due to suppressing the activation of the JAK/STAT signaling pathway. However, the specific and detailed mechanism by which RP11-468E2.5 negatively targets STAT5 and STAT6 remain unclear. Therefore, further investigation is warranted, aiming ultimately to develop a safer and more efficient method of IncRNA-targeting cancer diagnosis and therapy.

\section{Abbreviations \\ AJCC: American Joint Committee on Cancer; ANOVA: Analysis of variance; BCA: Bicinchoninic acid; BCl-2: B-cell lymphoma 2; BSA: Bovine serum albumin; CCND1: Cyclin D1; CDNA: complementary DNA; CRC: Colorectal cancer; DAB: Diaminobenzidine; DAPI: 4'6-diamidino-2-phenylindole; DMSO: Dimethylsulfoxide; ECL: Enhanced chemiluminescence; \\ ELISA: Enzyme-linked immunosorbent assay; FBS: Fetal bovine serum; FITC: Fluorescein isothiocyanate; GAPDH: glyceraldehyde-3-phosphate dehydrogenase; GEO: Gene Expression Omnibus; IgG: immunoglobulin G; JAK/STAT: Janus kinase-signal transducer and activator of transcription; KEGG: Kyoto Encyclopedia of Genes and Genomes; LncRNAs: Long non- coding RNAs; MTT: 3-(4,5-dimethylthiazol-2-yl)-2,5-diphenyl-tetrazolium bromide; NC: negative control; OD: Optical density; p: phosphorylated; PAGE: Polyacrylamide gel electrophoresis; PBS: Phosphate buffered saline; PBST: Phosphate buffered saline with Tween-20; PI: Propidium iodide; PMSF: Phenylmethylsulphonyl fluoride; PVDF: Polyvinylidene difluoride; RIP: RNA Binding protein immunoprecipitation: \\ RIPA: Radioimmunoprecipitation assay; RPMI: Roswell Park Memorial Institute; RT-qPCR: Reverse transcription quantitative polymerase chain reaction; siRNA: small interference RNA; TBST: Tris-buffered saline Tween-20; \\ TNM: Tumor node metastasis; WHO: World Health Organization}

\section{Acknowledgements}

We would like to give our sincere appreciation to the reviewers for their helpful comments on this article. 


\section{Authors' contributions}

Yin-Ling Mao, Xu-Hai Zhao, Jun-Feng Wang, Hui-Jun Zheng, Qing-Shan You and Li Jiang designed the study. Yin-Ling Mao, Xu-Hai Zhao and Jun-Feng Wang Li collated the data, Hui-Jun Zheng, Qing-Shan You and Li Jiang designed and developed the database, carried out data analyses and produced the initial draft of the manuscript. Yin-Ling Mao and Xu-Hai Zhao contributed to drafting the manuscript. All authors have read and approved the final submitted manuscript.

\section{Funding}

This study was supported by "Effects of long non-coding RNA ANRIL on proliferation, apoptosis and autophagy of diffuse large B-cell lymphoma cells via the NF-KB signaling pathway" (No. LH2019H101).

\section{Availability of data and materials}

The datasets generated during the current study are available.

\section{Ethics approval}

This study was performed with the approval from the Ethics Committee of the Harbin Medical University Tumour Hospital. All participating patients provided written informed consents. Animal experiments in this study were carried out in strict accordance with the Guide for the Care and Use of Laboratory animals published by the US National Institutes of Health.

\section{Consent for publication}

Not applicable.

\section{Competing interests}

The authors have declared that no competing interests exist.

\section{Author details}

'Department of Hematology and Lymphatic Diseases, Harbin Medical University Tumour Hospital, Harbin 150081, People's Republic of China. ${ }^{2}$ Department of Breast Surgery, Harbin Medical University Tumour Hospital, Harbin 150081, People's Republic of China. ${ }^{3}$ Department of Abdominal Radiotherapy, Harbin Medical University Tumour Hospital, No. 150, Haping Road, Nangang District, Harbin 150081, People's Republic of China.

${ }^{4}$ Department of Thoracic Surgery, Harbin Medical University Tumour Hospital, Harbin 150081, People's Republic of China. ${ }^{5}$ Department of General Surgery, Kangying Hospital of Mingshui County, Suihua 151700, People's Republic of China.

Received: 27 March 2019 Accepted: 23 September 2019

Published online: 12 November 2019

\section{References}

1. Wu C, Zhu X, Liu W, Ruan T, Tao K. Hedgehog signaling pathway in colorectal cancer: function, mechanism, and therapy. Onco Targets Ther. 2017;10:3249-59.

2. Atkin WS, Edwards R, Kralj-Hans I, Wooldrage K, Hart AR, Northover JM, et al. Once-only flexible sigmoidoscopy screening in prevention of colorectal cancer: a multicentre randomised controlled trial. Lancet. 2010;375(9726): 1624-33.

3. Li SQ, Su N, Gong P, Zhang HB, Liu J, Wang D, et al. The expression of Formyl peptide receptor 1 is correlated with tumor invasion of human colorectal Cancer. Sci Rep. 2017;7(1):5918.

4. Fan Q, Liu B. Identification of the anticancer effects of a novel proteasome inhibitor, ixazomib, on colorectal cancer using a combined method of microarray and bioinformatics analysis. Onco Targets Ther. 2017;10:3591-606.

5. Zhang YH, Fu J, Zhang ZJ, Ge CC, Yi Y. LncRNA-LINC00152 down-regulated by miR-376c-3p restricts viability and promotes apoptosis of colorectal cancer cells. Am J Transl Res. 2016;8(12):5286-97.

6. Brate J, Adamski M, Neumann RS, Shalchian-Tabrizi K, Adamska M. Regulatory RNA at the root of animals: dynamic expression of developmental lincRNAs in the calcisponge Sycon ciliatum. Proc Biol Sci. 2015;282(1821):20151746

7. O'Shea JJ, Schwartz DM, Villarino AV, Gadina M, Mclnnes IB, Laurence A. The JAK-STAT pathway: impact on human disease and therapeutic intervention. Annu Rev Med. 2015;66:311-28.

8. Reich NC. STATs get their move on. JAKSTAT. 2013;2(4):e27080.
9. Sehgal PB. Paradigm shifts in the cell biology of STAT signaling. Semin Cell Dev Biol. 2008;19(4):329-40.

10. Freund $P$, Kerenyi MA, Hager M, Wagner $T$, Wingelhofer B, Pham HTT, et al. O-GlcNAcylation of STAT5 controls tyrosine phosphorylation and oncogenic transcription in STAT5-dependent malignancies. Leukemia. 2017;31(10): 2132-42.

11. O'Shea JJ, Plenge R. JAK and STAT signaling molecules in immunoregulation and immune-mediated disease. Immunity. 2012;36(4):542-50.

12. Uchiyama T, Takahashi H, Sugiyama M, Sakai E, Endo H, Hosono K, et al. Leptin receptor is involved in STAT3 activation in human colorectal adenoma. Cancer Sci. 2011;102(2):367-72.

13. Liu Y, Guo R, Hao G, Xiao J, Bao Y, Zhou J, et al. The expression profiling and ontology analysis of noncoding RNAs in peritoneal fibrosis induced by peritoneal dialysis fluid. Gene. 2015;564(2):210-9.

14. Mao YL, Li ZW, Lou CJ, Pang D, Zhang YQ. Phospho-STAT5 expression is associated with poor prognosis of human colonic adenocarcinoma. Pathol Oncol Res. 2011;17(2):333-9.

15. Mao Y, Li Z, Lou C, Zhang Y. Expression of phosphorylated Stat5 predicts expression of cyclin D1 and correlates with poor prognosis of colonic adenocarcinoma. Int J Color Dis. 2011;26(1):29-35.

16. Fujita A, Sato JR, Rodrigues Lde O, Ferreira CE, Sogayar MC. Evaluating different methods of microarray data normalization. BMC Bioinformatics. $2006 ; 7(469)$

17. Smyth GK. Linear models and empirical bayes methods for assessing differential expression in microarray experiments. Stat Appl Genet Mol Biol. 2004;3(Article3).

18. Wang J, Duncan D, Shi Z, Zhang B. WEB-based GEne SeT AnaLysis Toolkit (WebGestalt): update 2013. Nucleic Acids Res. 2013;41(Web Server issue): W77-83.

19. Choi KH, Kim BS, Oh ST, Yook JH, Kim BS. Comparison the sixth and seventh editions of the AJCC staging system for T1 gastric cancer: a long-term follow-up study of 2124 patients. Gastric Cancer. 2017;20(1):43-8.

20. Chesworth BM, Hamilton CB, Walton DM, Benoit M, Blake TA, Bredy $\mathrm{H}$, et al. Reliability and validity of two versions of the upper extremity functional index. Physiother Can. 2014;66(3):243-53.

21. Smith DL, Comer TP. Gastrocolic fistula, a rare complication of benign gastric ulcer: report of a case. Dis Colon Rectum. 1974;17(6):769-70.

22. Staudacher JJ, Bauer J, Jana A, Tian J, Carroll T, Mancinelli G, et al. Activin signaling is an essential component of the TGF-beta induced pro-metastatic phenotype in colorectal cancer. Sci Rep. 2017;7(1):5569.

23. Liz J, Esteller M. IncRNAs and microRNAs with a role in cancer development. Biochim Biophys Acta. 2016;1859(1):169-76.

24. Slattery ML, Lundgreen A, Kadlubar SA, Bondurant KL, Wolff RK. JAK/STAT/ SOCS-signaling pathway and colon and rectal cancer. Mol Carcinog. 2013; 52(2):155-66.

25. Huang JZ, Chen M, Chen GXC, Zhu S, Huang H, et al. A peptide encoded by a putative IncRNA HOXB-AS3 suppresses Colon Cancer growth. Mol Cell. 2017;68(1):171-84 e6.

26. Damas ND, Marcatti M, Come C, Christensen LL, Nielsen MM, Baumgartner $\mathrm{R}$, et al. SNHG5 promotes colorectal cancer cell survival by counteracting STAU1-mediated mRNA destabilization. Nat Commun. 2016;7(13875).

27. Jiang $H$, Wang $Y, A i$ M, Wang $H$, Duan $Z$, Wang $H$, et al. Long noncoding RNA CRNDE stabilized by hnRNPUL2 accelerates cell proliferation and migration in colorectal carcinoma via activating Ras/MAPK signaling pathways. Cell Death Dis. 2017;8(6):e2862.

28. Sehgal PB. Non-genomic STAT5-dependent effects at the endoplasmic reticulum and Golgi apparatus and STAT6-GFP in mitochondria. JAKSTAT. 2013;2(4):e24860.

29. Fang Y, Gu X, Li Z, Xiang J, Chen Z. miR-449b inhibits the proliferation of SW1116 colon cancer stem cells through downregulation of CCND1 and E2F3 expression. Oncol Rep. 2013;30(1):399-406.

30. Huang CY, Tsai CW, Hsu CM, Chang WS, Shui HA, Bau DT. The significant association of CCND1 genotypes with colorectal cancer in Taiwan. Tumour Biol. 2015;36(8):6533-40.

31. Kambara H, Gunawardane L, Zebrowski E, Kostadinova L, Jobava R, Krokowski D, et al. Regulation of Interferon-Stimulated Gene BST2 by a IncRNA Transcribed from a Shared Bidirectional Promoter. Front Immunol. 2014;5(676.

32. Shi D, Zheng H, Zhuo C, Peng J, Li D, Xu Y, et al. Low expression of novel IncRNA RP11-462C24.1 suggests a biomarker of poor prognosis in colorectal cancer. Med Oncol. 2014;31(7):31. 
33. Kang K, Robinson GW, Hennighausen L. Comprehensive meta-analysis of Signal Transducers and Activators of Transcription (STAT) genomic binding patterns discerns cell-specific cis-regulatory modules. BMC Genomics. 2013;14(4).

34. Huang C, Yang G, Jiang T, Cao J, Huang KJ, Qiu ZJ. Down-regulation of STAT3 expression by vector-based small interfering RNA inhibits pancreatic cancer growth. World J Gastroenterol. 2011;17(25):2992-3001.

35. Han D, Zhang Y, Chen J, Hua G, Li J, Deng X, et al. Transcriptome analyses of differential gene expression in the bursa of Fabricius between Silky Fowl and White Leghorn. Sci Rep. 2017;7(45959.

36. Yang Q, Li M, Wang T, Xu H, Zang W, Zhao G. Effect of STAT5 silenced by siRNA on proliferation apoptosis and invasion of esophageal carcinoma cell line Eca-109. Diagn Pathol. 2013;8(132.

37. Zhang L, Li J, Li L, Zhang J, Wang X, Yang C, et al. IL-23 selectively promotes the metastasis of colorectal carcinoma cells with impaired Socs 3 expression via the STAT5 pathway. Carcinogenesis. 2014:35(6):1330-40.

38. Wang CG, Ye YJ, Yuan J, Liu FF, Zhang H, Wang S. EZH2 and STAT6 expression profiles are correlated with colorectal cancer stage and prognosis. World J Gastroenterol. 2010;16(19):2421-7.

39. Mao YL, ZXH WJF. Effects of Long Non-Coding RNA RP11-468E2. 5 on Cell Proliferation and Apoptosis in Colorectal Cancer Cells by Targeting STAT5 and STAT6 via the JAK/STAT Signaling Pathway. STAT Signaling Pathway. 2019.

40. Chen $P$, Huang $L$, Zhang $Y$, Qiao M, Yao W, Yuan $Y$. The antagonist of the JAK-1/STAT-1 signaling pathway improves the severity of ceruleinstimulated pancreatic injury via inhibition of NF-kappaB activity. Int J Mol Med. 2011;27(5):731-8.

41. Heltemes-Harris LM, Willette MJ, Ramsey LB, Qiu YH, Neeley ES, Zhang N, et al. Ebf1 or Pax5 haploinsufficiency synergizes with STAT5 activation to initiate acute lymphoblastic leukemia. J Exp Med. 2011;208(6):1135-49.

42. Xiong $H$, Zhang $Z G$, Tian $X Q$, Sun DF, Liang QC, Zhang YJ, et al. Inhibition of JAK1, 2/STAT3 signaling induces apoptosis, cell cycle arrest, and reduces tumor cell invasion in colorectal cancer cells. Neoplasia. 2008;10(3):287-97.

43. Lee $\mathrm{H}$, Li WP, Hisert KB, Ivashkiv LB. Inhibition of interleukin 2 signaling and signal transducer and activator of transcription (STAT) 5 activation during $T$ cell receptor-mediated feedback inhibition of T cell expansion. J Exp Med. 1999;190(9):1263-74.

44. Matsumura I, Kitamura T, Wakao H, Tanaka H, Hashimoto K, Albanese C, et al. Transcriptional regulation of the cyclin D1 promoter by STAT5: its involvement in cytokine-dependent growth of hematopoietic cells. EMBO J. 1999;18(5):1367-77.

45. Buitenhuis M, Coffer PJ, Koenderman L. Signal transducer and activator of transcription 5 (STAT5). Int J Biochem Cell Biol. 2004;36(11):2120-4.

46. Salmena L, Poliseno L, Tay Y, Kats L, Pandolfi PP. A ceRNA hypothesis: the Rosetta stone of a hidden RNA language? Cell. 2011;146(3):353-8.

\section{Publisher's Note}

Springer Nature remains neutral with regard to jurisdictional claims in published maps and institutional affiliations.

Ready to submit your research? Choose BMC and benefit from:

- fast, convenient online submission

- thorough peer review by experienced researchers in your field

- rapid publication on acceptance

- support for research data, including large and complex data types

- gold Open Access which fosters wider collaboration and increased citations

- maximum visibility for your research: over $100 \mathrm{M}$ website views per year

At $\mathrm{BMC}$, research is always in progress.

Learn more biomedcentral.com/submissions 